\title{
Evidências Preliminares da Efetividade do Treinamento Cognitivo para Melhorar a Inteligência de Crianças Escolares
}

\author{
Preliminary Evidence of Effectiveness in Cognitive Training \\ to Improve School Children Intelligence
}

\author{
Marcela Mansur-Alves*, Carmen Flores-Mendoza \& Carlos Julio Tierra-Criollo \\ Universidade Federal de Minas Gerais, Belo Horizonte, Minas Gerais, Brasil
}

\begin{abstract}
Resumo
Objetivou-se verificar a efetividade de um programa de treinamento cognitivo (TC) para melhorar a inteligência de crianças escolares. Participaram do TC 16 crianças $(M=8,75$ anos; $D P=0,44)$ divididas em grupo controle (GC) e grupo experimental (GE), avaliadas no pré e no pós-teste com medidas de inteligência fluida, cristalizada e desempenho escolar. O TC consistiu da aplicação de tarefas de memória de trabalho por dois meses. Não foram encontradas diferenças significativas entre as medidas pré e pós-teste de inteligência. Entretanto, após o treinamento, observou-se redução das diferenças entre $\mathrm{G} c$ e $\mathrm{G} f$ em medidas de inteligência fluída. No caso do teste Raven, a diferença entre GC e GE (favorável a GC) caiu de um $d=0,126$ para $d=0,058$. No teste TNVRI a diferença inicial a favor de GC $(d=0,177)$ passou a ser negativa $(d=-0,384)$, portanto a favor de GE. Esse pequeno benefício não foi observado nos testes de conhecimento escolar, nos quais os maiores ganhos no pós-teste foram do GC. Os resultados encontram suporte parcial na literatura. Contudo, estudos com uma amostra maior, maior tempo de treinamento e de intervalo entre os pós-testes são necessários para verificar a generalidade de tais achados.

Palavras-chave: Treinamento cognitivo, inteligência fluida, inteligência cristalizada.
\end{abstract}

\begin{abstract}
The present research intends to verify the effectiveness of a cognitive training (CT) to foster intelligence of school Brazilian children from different intellectual levels. Sixteen fourth-grade boys $(M=8.75$ years; $S D=.44)$ took part in this study and were randomly selected to the training group (TG) or control group (CG). In the pre and posttest, all children were assessed using measures with measures of fluid, crystallized intelligence, and school achievement. For the training program we used working memory task for a period of two months. Results: no statically significant difference was found between both groups at posttest in none of the measures used. However, after training, there was a reduction of the differences between GC and GF on measures of fluid intelligence. In the case of Raven, the difference between CG and GE (favorable to GC) dropped from $d=.126$ to .058 . In the TNVRI test, the initial difference in favor of CG $(d=.177)$ became negative $(d=-.384)$, therefore in favor of GE. This small benefit was not observed in tests of school knowledge, where GC had the biggest gains in the post-test assessment. The results are partially supported in the literature. However, studies with a larger sample, more training sessions and longer interval between post-tests to verify the generality of these findings.

Keywords: Cognitive training, fluid intelligence, crystallized intelligence.
\end{abstract}

A inteligência humana pode ser considerada um dos construtos mais investigados na historia da ciência psicológica em todos os tempos (Lubinski, 2004). A inteligência pode ser entendida como "a capacidade para entender idéias complexas, adaptar efetivamente ao ambiente, aprender a partir da experiência, engajar em várias formas

\footnotetext{
* Endereço para correspondência: Laboratório de Avaliação das Diferenças Individuais, Departamento de Psicologia, Faculdade de Filosofia e Ciências Humanas, Universidade Federal de Minas Gerais, Sala 4008, Av. Antônio Carlos, 6627, Campus Pampulha, Belo Horizonte, MG, Brasil 31270-901. E-mail: marcelamansuralves@ yahoo.com.br ou marmansura@gmail.com
}

de raciocínio e superar os obstáculos" (Neisser et al., 1996, p. 77). Não obstante possa haver ainda desacordos com relação às dimensões específicas e organização estrutural da inteligência, a grande maioria dos pesquisadores da área reconhece a importância da inteligência para o sucesso individual em vários aspectos da vida (Strenze, 2007).

Nesse sentido, existe muita informação sobre a estrutura, os determinantes e o curso do desenvolvimento da inteligência humana, mas pouco se sabe ainda sobre a possibilidade de modificação da mesma. Conforme ressaltava Jensen (1981), os psicólogos se esforçam para elaborar programas ou estratégias visando a modificação da capacidade intelectual dos indivíduos já desde o início 
do século XX. Na década de 1950, por exemplo, surgiram nos Estados Unidos da América (EUA) diversos programas de intervenção infantil destinados a crianças de ambiente social economicamente desvantajoso, baixo nível intelectual materno e com dificuldades desenvolvimentais, tais como o programa Head Start, o projeto Abecedário e o projeto Milwaukee (Gray \& McCormick, 2005; Ramey \& Ramey, 1998). Em geral, os principais resultados desses e outros programas de intervenção cognitiva que surgiram ao redor do mundo foram: (a) pequeno aumento de QI que não traria uma vantagem adaptativa real para a criança. Assim como, nenhum dos estudos teve o cuidado de verificar se o aumento de QI acontece em todos os níveis de QI inicial; (b) consistente "fade-out effects", isto é, diminuição drástica ou perda total dos ganhos cognitivos ao longo do tempo; (c) efeito mais acentuado em habilidades cognitivas específicas do que em capacidades gerais (Campbell et al., 2002; Colom, 2008; Herrnstein \& Murray, 1994). Assim, a falha em encontrar resultados positivos e vantajosos para os participantes de treinamentos cognitivos tem gerado além de desmotivação acadêmica, constante debate e controvérsias.

Nesse sentido, até poucos anos atrás, a conclusão geral da comunidade acadêmica era a de que as intervenções existentes tinham pouco ou nenhum sucesso para a modificação intelectual (Buschkuehl \& Jaeggi, 2010). Entretanto, nos últimos anos, um número significativo de estudos tem sido publicado mostrando que certas intervenções possuem um impacto positivo na inteligência (Nisbett, 2009). Entre essas intervenções estão aquelas relacionadas à memória de trabalho (MT) como veículo de mudança na inteligência. A memória de trabalho é entendida como um sistema cognitivo que armazena e manipula uma quantidade limitada de informação em um período curto de tempo (Baddeley, 2003). Existem evidências nacionais e internacionais de que a MT é essencial para o desempenho de tarefas que envolvam funções cognitivas superiores, tais como leitura, raciocínio e resolução de problemas (Flores-Mendoza, Colom, Garcia, \& Castilho, 2001; Jaeggi, Buschkuehl, Jonides, \& Shah, 2011). Tais evidências não são surpreendentes se se considera que a memória de trabalho parece ser o melhor preditor individual da capacidade de raciocínio e da inteligência geral (Ackerman, Beier, \& Boyle, 2005; Colom \& Flores-Mendoza, 2001; Conway, Kane, \& Engle, 2003; Kane \& Engle, 2002; Kyllonen \& Christal, 1990). Ademais, Kane e Engle (2002) chamam atenção para o fato de que a MT e a inteligência geral compartilham circuitos cerebrais semelhantes. Mais especificamente, os autores chamam a atenção para o modelo proposto por Cohen e colegas (Miller \& Cohen, 2001) que destaca a função do córtex pré-frontal (PF), do cingulado anterior e do hipocampo em manter a informação contextual em estado ativo, além de atuar na redução de distratores através de inibição lateral e processamento dinâmico, funções essas bastante semelhantes àquelas do sistema de MT e da inteligência. Portanto, o treinamento de memória de trabalho pode possuir impacto positivo na promoção de mudanças nas capacidades de leitura e de raciocínio (Klingberg, 2010), na medida em que ambos os sistemas (MT e inteligência) estariam assentados em circuitos cerebrais semelhantes, embora se ressalte que os construtos não são idênticos (Ackerman et al., 2005; Garlick, 2002; Kane \& Engle, 2002). A semelhança entre esses construtos seria um fator essencial para a elaboração de treinamentos bem-sucedidos, conforme destacam Barnett e Ceci (2002), pois facilitam a transferência dos ganhos.

Um dos primeiros trabalhos utilizando programas de MT para melhorar a inteligência foi publicado por Klingberg, Forssberg e Westerber (2002), no qual demonstraram o valor terapêutico de um treino de MT no tratamento do Transtorno do Déficit de Atenção/Hiperatividade (TDAH). Quatorze crianças com idade média de onze anos, diagnosticadas com o TDAH, foram divididas em um grupo de treinamento, o qual recebeu o treinamento por um período de 5-6 semanas, e um grupo controle sem contato. Os autores utilizaram três tarefas simples de memória de trabalho e uma tarefa de tempo de reação. As crianças participantes do grupo de treinamento apresentaram ganhos significativos no desempenho no teste Raven comparando o pré com o pós-teste. Em um segundo experimento, Klingberg et al. (2002) treinaram quatro adultos jovens (idades entre 23 e 29 anos) saudáveis utilizando o mesmo regime de treinamento, não sendo utilizado um grupo de controle. Resultados semelhantes foram encontrados, ou seja, aumento do desempenho intelectual após o treinamento.

Klingberg et al. (2005) tentaram estender os achados anteriores a uma amostra maior (44 crianças com TDAH e idade média de dez anos), sendo 20 do grupo experimental e 24 do controle. Os autores realizaram, ainda, um segundo pós-teste três meses após o término do treino. Tanto no pós-teste imediato quanto no segundo pós-teste, os resultados encontrados replicam o dos estudos anteriores, isto é, o grupo de treinamento apresentando melhor desempenho no teste Raven do que o controle. Em um novo estudo com pré-escolares saudáveis, o grupo de Klingberg (Thorell, Lindqvist, Bergman, Bohlin, \& Klingberg, 2009) usou o mesmo protocolo de treinamento empregado no estudo de 2005, mas não foram observadas melhoras no desempenho do grupo de treinamento comparado ao controle na Escala Wechsler de Inteligência para Pré-escolares. Similarmente, Holmes, Gathercole e Dunnings (2009) não conseguiram replicar as melhoras cognitivas em crianças com MT reduzida usando o mesmo programa de treinamento proposto por Klingberg et al. (2002).

Jaeggi, Buschkuehl, Jonides e Perrig (2008) demonstraram a efetividade do treinamento em MT para o aumento da inteligência fluida $(\mathrm{G} f)$ de adultos universitários. Setenta universitárias saudáveis ( $M=25,6$ anos; $D P=3,3$ anos) foram divididas em um grupo de intervenção e um grupo controle sem contato. O teste Matrizes Progressivas de Raven - Escala Avançada ou o Teste de Matrizes de Bochumer (BOMAT) foram utilizados para avaliar os ganhos em $\mathrm{G} f$. O grupo de intervenção foi subdivido em quatro de acordo com o número de sessões de treinamento (oito, 
Mansur-Alves, M., Flores-Mendoza, C. \& Tierra-Criollo, C. J. (2013). Evidências Preliminares da Efetividade do Treinamento Cognitivo para Melhorar a Inteligência de Crianças Escolares.

doze, dezessete e dezenove dias). Cada sessão teve duração de 25 minutos e a tarefa de MT utilizada foi a $n$-back, que consiste na apresentação de fileiras de estímulo uma após a outra. Em cada apresentação em que o estímulo atual é o mesmo que foi apresentado $n$ tentativas anteriores, o participante é solicitado a apertar um botão. Os autores utilizaram a versão dual para o estudo, na qual os participantes precisam desempenhar a tarefa simultaneamente em duas modalidades sensoriais (visual e auditiva). Os resultados encontrados mostraram que tanto o grupo controle quanto o experimental aumentaram seus escores no teste de inteligência, contudo o aumento para o grupo experimental foi significantemente maior do que para o controle ( $d=0,65$ e $d=0,25$, respectivamente). Ademais, diferenças significativas foram encontradas entre os grupos de treinamento de acordo com o número de sessões de treinamento. Moody (2009) recomenda cautela ao interpretar os achados de Jaeggi et al. (2008), destacando que o trabalho sofre de alguns problemas metodológicos. Por exemplo, Moody (2009) destaca que os itens tanto do Raven quanto do BOMAT são organizados em ordem crescente de dificuldade e que, usualmente, o sujeito tem cerca de 50 minutos para realizar os testes. Contudo, nos estudos acima relatados, os pesquisadores deram apenas 10 minutos para os participantes terminaram o BOMAT, significando que os mesmos não tiveram oportunidade de realizar os itens mais difíceis do teste. Segundo Moody (2009), a restrição de tempo colocada no teste alterou a finalidade do instrumento e, portanto, comprometeu a validade interna do estudo.

$\mathrm{Na}$ tentativa de verificar a pertinência das críticas sugeridas por Moody (2009), Colom et al. (2010) realizaram estudo semelhante ao de Jaeggi et al. (2008). Duzentos e oitenta e oito participantes $(82 \%$ de mulheres, com idade média $=20,1$ anos e $D P=3,4$ anos) completaram três semanas de treinamento cognitivo. A capacidade cognitiva de todos os participantes foi avaliada com as Matrizes Progressivas de Raven - Escala Avançada e com os subtestes de raciocínio abstrato, verbal e relações espaciais da Bateria Diferencial de Aptidões (DAT) no pré e no pós-teste, os quais fora aplicados sem limite de tempo. A amostra foi dividida, então, em dois grupos: 173 participantes realizaram o treinamento com tarefas de memória de curto-prazo e MT, enquanto 115 participantes realizaram tarefas de velocidade de processamento e atenção. Os principais resultados do estudo revelam aumento dos escores nos testes de inteligência do pré-teste para o pós-teste (mais de um desvio-padrão, em média), resultado semelhante ao encontrado por Jaeggi et al. (2008). Entretanto, não houve diferença nos ganhos alcançados entre o grupo que realizou treinamento em MT e aquele que fez somente tarefas de velocidade de processamento e atencionais. Segundo Colom et al. (2010), esse resultado indica que os treinamentos de memória de trabalho não são únicos em produzir alterações na inteligência.

Já Alloway e Alloway (2009) utilizam o treinamento em MT para promover melhoras na inteligência crista- lizada. Quinze crianças ( $M=12,9$ anos; $D P=0,4$ anos) com dificuldades de aprendizagem realizaram medidas de conhecimento geral no pré e pós-teste. Oito crianças foram submetidas ao treinamento de MT com tarefas verbais e visuo-espaciais. $\mathrm{O}$ grupo controle participou de atividades de aprendizagem dirigida. Relativo ao grupo controle, o grupo de treinamento teve altos escores nas medidas de conhecimento geral após oito semanas de treinamento (diferença entre os grupos foi de 10 pontos de QI, $p<$ 0,05). Em estudo mais recente, Chein e Morrison (2010) não encontraram aumento do desempenho no teste Raven Avançado em adultos jovens após treinamento em MT.

Em geral, os resultados apresentados são mistos. Alguns estudos fracassaram em encontrar resultados positivos, mas a maior parte dos estudos mostrou resultados favoráveis à intervenção utilizando a MT. Shipstead, Redick e Engle (2010) acrescentam que os estudos que utilizam a MT como veículo de mudança intelectual são, ainda, recentes e carecem, portanto, de cuidados metodológicos adequados. Esses autores destacam, por exemplo, a necessidade de se utilizar uma bateria de testes de inteligência, ao invés de um instrumento único, para se verificar a melhora após treinamento. Adicionalmente pode-se citar que nenhum dos estudos controla o QI inicial dos participantes. Não se sabe, portanto, quais os níveis de inteligência mais suscetíveis à modificação.

Apesar dos problemas metodológicos, Shipstead et al. (2010) apontam a MT como a estratégia de intervenção mais promissora encontrada nos últimos anos. No Brasil, não foi encontrado nenhum estudo de treinamento cognitivo utilizando MT como foco de treinamento. Encontram-se sim alguns poucos estudos, muito antigos, utilizando tarefas psicomotoras (ver, por exemplo, o estudo de Dantas, 1979). Nesse sentido, a presente pesquisa objetiva verificar a efetividade de um programa de treinamento cognitivo utilizando a memória de trabalho na melhora da inteligência de crianças escolares da cidade de Belo Horizonte.

\section{Método}

\section{Participantes}

Primeiramente selecionou-se 124 alunos cursando o quarto e quinto ano do $2^{\circ}$ ciclo escolar de uma escola pública federal da cidade de Belo Horizonte, $54 \%$ do sexo masculino, com média de 9,19 anos ( $\mathrm{DP}=0,67$ ). Embora a idade "ótima" para treinamento cognitivo seja ainda desconhecida, alguns estudos sugerem que dois critérios associados são importantes para maior eficácia das estratégias interventivas - maior plasticidade cerebral (menor idade) e presença de certo nível de maturidade das estruturas relacionadas às funções de interesse (Nutley et al., 2011; Thompson-Schill, Ramscar, \& Chrysikou, 2009). Nesse sentido, no presente trabalho, a faixa etária escolhida contrabalançou o critério de menor idade (maior plasticidade) com a fase do desenvolvimento em que já se observa maturidade dos lobos frontais e de suas funções relacionadas (Garlick, 2002). 
Os resultados de dois testes de inteligência (Raven Escala Geral e TNVRI) foram utilizados para selecionar os participantes do treinamento cognitivo. Assim, as pontuações brutas de cada um dos testes foram transformadas a escore $z$, controlando a idade e o sexo, haja vista o efeito desenvolvimental esperado. Depois, os escores $z$ para cada teste foram transformados a QI (pela fórmula $z^{*} 15+100$ ) e esses foram categorizados em três intervalos: QI alto (pontuação > 120), QI médio (pontuação entre 95-110), e QI baixo (pontuação entre 70-85). Logo em seguida, foi realizado o procedimento de tabulação cruzada entre os QIs encontrados nos dois testes de inteligência a fim de verificar se a posição da criança segundo seu nível intelectual se mantinha a mesma nos três instrumentos. Assim, foram selecionadas apenas as crianças classificadas no mesmo intervalo de QI para os dois testes. Apenas meninos foram selecionados para participar do treinamento na tentativa de eliminar as diferenças cognitivas, motivacionais e de personalidade relacionadas a essa variável e que são constantemente apontadas na literatura (Halpern, 1997).

Com base nos critérios definidos acima, a amostra final seria constituída de 27 meninos (sete de QI baixo, 14 de QI médio e seis de QI alto) selecionados para o estudo. Não obstante, caso a criança viesse a apresentar sintomas/diagnóstico de transtornos de aprendizagem, deficiência mental e dificuldades de atenção ela seria excluída do estudo, já que se pretendia trabalhar com uma amostra não clínica. As informações sobre a saúde da criança concedidas pelos pais por meio do Questionário de Saúde e informações cedidas pelo Serviço de Saúde da escola permitiram inferir que todos eles eram clinicamente saudáveis, sendo, portanto, incluídas no estudo.

Entretanto, devido a que alguns pais não consentiram a participação de seus filhos no estudo, a amostra final resultou em 16 meninos (59\% dos participantes), sendo quatro de QI baixo, quatro de QI médio e quatro de QI alto. Os desistentes não diferiram dos demais em termos de idade, nível socioeconômico e escolaridade dos pais. O agrupamento das crianças em controle (GC) e experimental (GE) foi feita de forma aleatória, sendo oito meninos para o GC e oito para GE. A idade média dos meninos selecionados foi de 8,75 anos, com desvio-padrão de 0,44 , sendo todos do $4^{\circ}$ ano do segundo ciclo. Não foram encontradas diferenças estatisticamente significativas entre GE e GC no que diz respeito à idade, nível de escolaridade dos pais, nível socioeconômico da família e saúde geral $(p>0,05)$

\section{Instrumentos}

Os instrumentos de medição cognitiva foram selecionados de forma a abranger diversos aspectos da inteligência humana, superando com isso a limitação de estudos anteriores em que se utilizou apenas uma medida cognitiva. Para o presente estudo os instrumentos utilizados foram aqueles considerados como representantes de inteligência fluída, isto é, medidas cognitivas que dependem pouco da aprendizagem escolar; e medidas de inteligência cristalizada, isto é, medidas que dependem da instrução escolar. Foram eles:
Matrizes Progressivas de Raven - Escala Geral (Centro Editor de Psicologia Aplicada [CEPA], 2001a). É uma medida de inteligência não-verbal associada à avaliação da inteligência fluída. Apresenta 60 itens distribuídos em cinco séries de 12 itens cada uma. Sua consistência interna para o presente estudo foi de 0,92 .

Teste Não-Verbal de Raciocínio Infantil (TNVRI; Pasquali, 2005). Avalia a inteligência geral (ou inteligência fluída) na medida em que foi construído com base nas Matrizes Progressivas de Raven. Possui 58 itens divididos em cinco séries com níveis de dificuldade crescentes. A tarefa do examinando consiste em encontrar o pedaço que está faltando em cada um dos desenhos apresentados. O índice de consistência interna para o presente estudo foi de 0,91 .

Bateria Fatorial CEPA (CEPA, 2001b). Foram utilizados os subtestes de fluência verbal, sinônimos, rapidez e exatidão de cálculos e séries numéricas. O teste de Sinônimos é utilizado para medir a compreensão da linguagem e compõe-se de 60 itens, nos quais são apresentados grupos de palavras. A tarefa do sujeito é escolher a palavra do grupo que possui o mesmo significado da palavra destacada. No teste de Fluência Verbal, o participante deve escrever o maior número de palavras que começa com uma determinada letra do alfabeto. O número total de palavras escritas corretamente e sem repetição representa o escore total do sujeito. O teste de Rapidez e Exatidão de Cálculos constitui-se de 25 adições e 15 multiplicações. Requer do examinando certo automatismo no calculo de operações fundamentais. E o teste de Séries Numéricas é uma medida de raciocínio abstrato. Ele é composto de 20 séries numéricas cujo último número da série está ausente. Todas as medidas são aplicadas com tempo de cinco minutos, após o qual a aplicação é interrompida. No presente estudo, as correlações entre os subtestes variaram entre 0,192 (fluência verbal e exatidão de cálculos) e 0,499 (cálculo e séries numéricas).

Teste de Desempenho Escolar (Stein, 1994). O instrumento é composto por três subtestes: Escrita, sob a forma de ditado de palavras isoladas (35 itens); Aritmética, com resoluções de operações matemáticas (38 itens), e o de Leitura para reconhecimento de palavras (70 itens). Os itens são dispostos de acordo com grau de dificuldade. A aplicação é individual. O instrumento apresenta bons índices de precisão. As correlações entre TDE com as medidas cognitivas foram todas significativas e acima de 0,35 .

Critério de Classificação Econômica Brasil (CCEB; Associação Brasileira de Empresas de Pesquisa [ABEP], 2003). Busca averiguar junto às famílias os bens que elas possuem e em que quantidade. Entre esses bens tem-se: automóvel, geladeira, televisão, dentre outros. Além disso, é questionado o nível de instrução do principal provedor da família. A partir da pontuação na escala de itens de consumo obtêm-se a classificação econômica que, no Brasil, se distribui em sete classes: A1, A2, B1, B2, C1, C2, D e E.

Questionário de Saúde. O questionário, elaborado especialmente para a presente pesquisa é composto por itens que avaliam a presença de problemas de saúde na 
Mansur-Alves, M., Flores-Mendoza, C. \& Tierra-Criollo, C. J. (2013). Evidências Preliminares da Efetividade do Treinamento Cognitivo para Melhorar a Inteligência de Crianças Escolares.

criança, tais como transtornos de aprendizagem, ansiedade, dificuldades de atenção e dificuldades físicas, e se a mesma faz uso de alguma medicação controlada. Serviu como referência para um dos critérios de exclusão do estudo, a saber, a presença de transtornos de aprendizagem, deficiência mental, dificuldades de atenção, haja vista se tratar de um estudo com amostra não clínica.

\section{Procedimento}

O projeto foi aprovado pelo Comitê de Ética em Pesquisa (COEP) da Universidade Federal de Minas Gerais (UFMG) em novembro de 2009 (Parecer no. ETIC 0490.0.0.203.000-09). Os procedimentos desta pesquisa estão divididos em três etapas, a saber:

Etapa 1. Seleção da Amostra e Pré-Teste. Entre os meses de março e maio de 2010, as 124 crianças foram avaliadas com os testes Matrizes Progressivas de Raven Escala Geral, TNVRI e a Bateria CEPA. As medidas foram administradas de forma coletiva nas salas de aula por estudantes de psicologia da UFMG treinados especialmente para o estudo. As sessões de aplicação tiveram duração de 2 horas e foram realizadas em dois dias, sendo que na primeira sessão aplicou-se o Raven e na segunda, o TNVRI e a Bateria CEPA. Delas, o Raven e o TNVRI serviram primeiramente como medidas de seleção de amostra. Já O TDE foi aplicado de forma individual apenas nas dezesseis crianças autorizadas a participar do treinamento cognitivo. A aplicação do TDE teve duração aproximada de 30 minutos com cada criança. Utilizaram-se, portanto, como medidas de pré-teste: o Raven, o TNVRI, a Bateria CEPA e o TDE.

Paralelamente foram enviados aos pais o questionário Critério Brasil e o Questionário de Saúde com solicitação de informações relativas à caracterização sociodemográfica e de saúde dos participantes. Ambos os questionários foram enviados em envelope lacrado pela escola e devolvidos pelos pais à mesma.

Etapa 2. Treinamento Cognitivo. Esta etapa teve início na segunda semana de agosto de 2010 e encerrou-se na última semana de outubro de 2010 (três meses). O treinamento cognitivo aconteceu nas dependências da escola em salas equipadas com computadores, nos horários de aula dos alunos. As crianças foram acompanhadas durante as sessões por quatro auxiliares de pesquisa, estudantes do sétimo e oitavo períodos do curso de psicologia da UFMG, as quais foram extensivamente treinadas no protocolo de intervenção utilizado. Faz-se importante notar que as auxiliares não tinham conhecimento do nível intelectual de cada uma das crianças participantes do treinamento.

As crianças, tanto de GE quanto de GC, foram divididas em grupos menores para facilitar a logística da intervenção e melhorar a supervisão das sessões. Assim, foram dois grupos controle e dois experimentais com três crianças cada e um grupo controle e um experimental com duas crianças cada. Cada grupo participou do treinamento duas vezes por semana em dias e horários alternados. O tempo de duração de cada sessão para o grupo experimental variou entre 40 e 50 minutos, dependendo do ritmo da criança. Para o grupo controle, as sessões tiveram duração fixa de 50 minutos.

As crianças do grupo experimental foram treinadas utilizando as tarefas informatizadas de MT, as quais foram adaptadas do software desenvolvido por Flores-Mendoza et al. (2001) para avaliação da MT em adultos com deficiência mental. Foram utilizadas três tarefas para treinamento. A tarefa Ordem Numérica é composta por quatro níveis de dificuldade (baixo, médio 1, médio 2, alto). Cada nível de dificuldade é composto por 30 ensaios e dois itens de treino. A criança avança de um nível para outro automaticamente quando atinge o critério de avanço especificado (nesse caso, $80 \%$ de acerto). A tarefa da criança consiste em realizar operações matemáticas de adição simples (por exemplo: $1+1=$ ? e $4+3$ $=$ ?) enquanto memoriza o resultado das mesmas, o qual deve ser colocado em ordem crescente. A cada nível o número de valores a ser memorizado aumenta. A tarefa $\mathrm{ABC}$ gramatical compõe-se de três níveis de dificuldade (baixo, médio e alto), sendo que cada nível contém 30 ensaios e dois itens de treino. A criança avança de um nível para outro automaticamente quando atinge o critério de avanço especificado (nesse caso, $80 \%$ de acerto). A tarefa consiste na apresentação de uma série de sentenças sobre a posição das letras, na qual a criança deverá responder qual a ordem corretas das letras apresentadas. Por exemplo: "A vem antes de B" (Tela 1); "Qual a ordem correta?: 1-BA 2- AB" (Tela 2). A cada nível, as sentenças vão se tornando mais complexas. A tarefa Alfabeto é composta de três níveis de dificuldade (baixo, médio e alto), sendo que cada nível apresenta 32 ensaios e dois itens de treino. A criança avança de um nível para outro automaticamente quando atinge o critério de avanço especificado (nesse caso, $80 \%$ de acerto). A tarefa consiste na apresentação de uma série de palavras, das quais a criança deverá lembrar a primeira letra e digitá-la em ordem alfabética (por exemplo: $\mathrm{MAU}, \mathrm{BAR}$ e $\mathrm{CEU}=\mathrm{B}, \mathrm{C}, \mathrm{M}$ ).

Já as crianças do grupo controle (GC), apesar de não receberem o treinamento cognitivo em MT, saíram de sala para realizar sessões de jogos livres (videogame), também no computador. Os jogos do grupo controle foram selecionados por não envolverem diretamente a memória de trabalho. A corrida de carros e o pinball são jogos que demandam mais controle motor do que outras habilidades (controle das teclas de direção no teclado para acelerar, freiar e posicionar o carro na pista de corrida e também para manter a bola na tela no jogo pinball). $\mathrm{O}$ tetris, embora também envolva controle motor, demanda maior nível de atenção do participante. O impacto da seleção desses jogos para realização das atividades do grupo controle nos resultados do estudo será discutido posteriormente.

Etapa 3. Pós-Teste. Na etapa de pós-teste, que ocorreu entre os meses de outubro e novembro de 2010, as crianças participantes do estudo (GC e GE) foram novamente submetidas aos testes Matrizes Progressivas de RavenEscala Geral, TNVRI, Bateria CEPA e TDE. De maneira 
semelhante ao pré-teste, o teste Raven, TNVRI e Bateria CEPA foram aplicados coletivamente nas salas de aula por estudantes de psicologia da UFMG. Foram duas sessões de aplicação de testes coletivos: na primeira delas, aplicou-se o Raven (duração de cerca de 50 minutos) e na segunda, aplicou-se o TNVRI e a Bateria CEPA com duração de 1 hora. O TDE foi aplicado individualmente. O período de pós-teste ocorreu um mês após o término da intervenção por questões de logística do estudo.

\section{Resultados}

Efeito do Treinamento em GC e GE para a Amostra Total (QI alto, médio e baixo)

Duas formas de análise foram consideradas. Em primeiro lugar, uma análise de variância de medidas repetidas intra e entre grupos é o teste estatístico aconselhável quando não existem diferenças significativas entre GC e GE no ponto de partida. Dessa forma se verifica se o ganho (ou perda) de escore após o treinamento é significativo (análise intragrupo) e se os ganhos (ou perdas) após o treinamento são diferentes entre os grupos (análise intergrupo). Em segundo lugar, se o ponto de partida é diferente para os grupos, caberia estimar um índice $d$ de Cohen ${ }^{1}$ para comparação de grupos antes e depois do treinamento cognitivo e assim identificar se ocorreu aumento ou redução das diferenças. Como pode ser observado na Tabela 1, antes do treinamento, o GC apresentava uma média maior que o GE nos testes de inteligência fluida Raven e TNVRI e uma média menor nas medidas de inteligência cristalizada (CEPA) e de desempenho escolar (TDE). Portanto, a seguir se apresentarão os dois tipos de análises estatísticas.

Tabela 1

Médias e Desvios-Padrões nos Testes Raven, TNVRI, CEPA e TDE, Antes e Depois do Treinamento para a Amostra Total $(N=16)$

\begin{tabular}{|c|c|c|c|}
\hline Grupo & Antes & Depois & $n$ \\
\hline \multicolumn{4}{|c|}{ Raven } \\
\hline Controle & $34,0(11,5)$ & $34,1(12,0)$ & 8 \\
\hline Experimental & $\begin{array}{c}32,5(12,2) \\
d=0,126\end{array}$ & $\begin{array}{c}33,4(12,2) \\
d=0,058\end{array}$ & 8 \\
\hline \multicolumn{4}{|c|}{ TNVRI } \\
\hline $\begin{array}{c}\text { Controle } \\
\text { Experimental }\end{array}$ & $\begin{array}{c}39,1(9,2) \\
37,1(13,1) \\
d=0,177\end{array}$ & $\begin{array}{c}33,7(13,8) \\
38,6(11,6) \\
d=-0,384\end{array}$ & $\begin{array}{l}8 \\
8\end{array}$ \\
\hline \multicolumn{4}{|c|}{ CEPA TOTAL } \\
\hline Controle & $35,1(12,1)$ & $53,8(12,9)$ & 8 \\
\hline Experimental & $\begin{array}{c}36,0(10,7) \\
d=-0,078\end{array}$ & $\begin{array}{c}40,4(12,6) \\
d=1,05\end{array}$ & 8 \\
\hline \multicolumn{4}{|c|}{ TDE } \\
\hline Controle & $99,6(14,1)$ & $110,5(9,5)$ & 8 \\
\hline Experimental & $\begin{array}{l}101,1(8,4) \\
d=-0,129\end{array}$ & $\begin{array}{c}109,7(10,2) \\
d=1,21\end{array}$ & 8 \\
\hline
\end{tabular}

Nota. * Os desvios-padrão estão entre parênteses; a bateria CEPA total representa a somatória dos escores dos testes de Fluência, Sinônimos, Cálculos e Séries Numéricas.

As análises de medidas repetidas intra e inter-grupo mostraram não haver efeito significativo do treinamento cognitivo para nenhum dos testes cognitivos e de desempenho escolar, como indicado pela significância de Wilks' $\Lambda$ utilizado para verificar o efeito de tempo (antes $\mathrm{x}$ depois). Os resultados evidenciaram não haver efeito de tempo, para nenhum dos dois grupos (controle e experimental), nos resultados do teste Raven $(\Lambda=0,99 ; F=0,065 ; p=$ $0,802)$. Não houve, também, efeitos diferenciados entre os grupos $(p>0,05)$. No TNVRI, os resultados encontrados evidenciaram não haver efeito de tempo, para nenhum dos dois grupos $(\Lambda=0,896 ; F=1,502 ; p=0,242 ; \eta 2=0,104)$.
Não houve, também, efeitos diferenciados entre os grupos $(p>0,05)$. Já para a CEPA, os resultados evidenciaram haver diferenças intra-grupos $(\Lambda=0,482 ; F=11,811 ; p=$ $0,006 ; \eta 2=0,518)$, ou seja, as médias de desempenho se alteraram do pré-teste para o pós-teste para cada grupo (GE e GC) separadamente. O mesmo aconteceu para o TDE $(\Lambda$ $=0,344 ; F=23,726 ; p=0,001 ; \eta 2=0,656)$. Contudo, não foram encontradas diferenças significativas entre os grupos $(p>0,05)$ tanto para a bateria CEPA quanto para o TDE.

${ }^{1}$ Ver fórmula para cálculo do índice $d$ no final do artigo. 
Mansur-Alves, M., Flores-Mendoza, C. \& Tierra-Criollo, C. J. (2013). Evidências Preliminares da Efetividade do Treinamento Cognitivo para Melhorar a Inteligência de Crianças Escolares.

Quando se estimou a diferença entre GC e GE no Raven, encontrou-se que o índice $d$ (favorável para GC) caiu de 0,126 (ou $0,126 \times 15=1,88$ pontos de QI) para $d=0,058$ (ou 0,057 x $15=0,86$ pontos de QI). No teste TNVRI, o índice $d$ favorável a GC era igual a 0,177 (ou 2,65 pontos de QI), mas após o treinamento a diferença foi favorável a GE ( $d=-0,384$ ou 5,7 pontos de QI). Portanto, nas medidas de inteligência fluída, o GE obteve um pequeno benefício, embora não significativo, em relação ao GC.

Contudo, nas medidas de inteligência cristalizada e de desempenho escolar a diferença continuou se mantendo favorável a GC conforme pode ser vista na Tabela 1. Tal resultado indicava que o GE não obteve nenhum benefício realmente significativo na esfera da aprendizagem.

\section{Efeito do Treinamento em GC e GE, Comparando QI Médio e Baixo}

Com relação ao efeito do treinamento, considerando apenas os grupos de QI médio e baixo para as análises, a Tabela 2 mostra as estatísticas descritivas antes e depois do treinamento em MT.

Tabela 2

Médias e Desvios-Padrões nos Testes Raven, TNVRI, CEPA e TDE, Antes e Depois do Treinamento para as Crianças de QI Médio e Baixo $(N=12)$

\begin{tabular}{|c|c|c|c|}
\hline Grupo & Antes & Depois & $n$ \\
\hline \multicolumn{4}{|c|}{ Raven } \\
\hline Controle & $29,3(9,0)$ & $28,6(9,0)$ & 6 \\
\hline Experimental & $27,6(9,9)$ & $31,8(11,8)$ & 6 \\
\hline & $d=0,179$ & $d=-0,31$ & \\
\hline \multicolumn{4}{|c|}{ TNVRI } \\
\hline Controle & $35,0(6,1)$ & $28,8(13,3)$ & 6 \\
\hline Experimental & $33,5(13,3)$ & $35,1(11,5)$ & 6 \\
\hline & $d=0,145$ & $d=-0,51$ & \\
\hline \multicolumn{4}{|c|}{ CEPA TOTAL } \\
\hline Controle & $32,6(9,7)$ & $49,2(6,9)$ & 6 \\
\hline Experimental & $34,0(11,9)$ & $34,6(9,5)$ & 6 \\
\hline & $d=-0,129$ & $d=1,758$ & \\
\hline \multicolumn{4}{|c|}{ TDE } \\
\hline Controle & $93,6(10,1)$ & $107,0(8,2)$ & 6 \\
\hline Experimental & $99,3(6,3)$ & $107,5(9,6)$ & 6 \\
\hline & $d=-0,129$ & $d=1,758$ & \\
\hline
\end{tabular}

Nota. * Os desvios-padrões estão entre parênteses; a bateria CEPA total representa a somatória dos escores dos testes de Fluência, Sinônimos, Cálculos e Séries Numéricas.

Novamente, considerando apenas as crianças de QI médio e baixo, as análises de medidas repetidas intra $\mathrm{e}$ inter-grupo mostram não haver efeito significativo do treinamento cognitivo para nenhum dos testes cognitivos e de desempenho escolar. Os resultados evidenciaram não haver efeito de tempo intra-grupos nos resultados do teste Raven $(\Lambda=0,763 ; F=2,792 ; p=0,129 ; \eta 2=0,237)$. Não houve, também, efeitos diferenciados entre os grupos ( $p$ $>0,05)$. No TNVRI, os resultados encontrados mostraram nos haver efeito de tempo intra-grupos $(\Lambda=0,932 ; F=$ $0,657 ; p=0,439 ; \eta 2=0,068)$. Não houve, também, efeitos diferenciados entre os grupos $(p>0,05)$. Já para a CEPA, os resultados evidenciaram haver diferenças intra-grupos $(\Lambda=0,598 ; F=5,370 ; p=0,049 ; \eta 2=0,402)$, ou seja, as médias de desempenho se alteraram do pré-teste para o pós-teste para cada grupo (GE e GC) separadamente. O mesmo aconteceu para o $\operatorname{TDE}(\Lambda=0,301 ; F=23,183$ $p=0,001 ; \eta 2=0,669)$. Contudo, não foram encontradas diferenças significativas entre os grupos $(p>0,05)$ tanto para a bateria CEPA quanto para o TDE.

Quando se considerou as diferenças entre GC e GE nos testes de inteligência fluida (Raven e TNVRI), os ganhos cognitivos foram maiores para GE do que para GC. Assim, no teste Raven o índice $d$ inicialmente favorável a GC ( $d=0,179$ ou 2,7 pontos de QI) se torna favorável a GE após o treinamento $(d=-0,31$ ou 4,6 pontos de QI). Igualmente, no teste TNVRI, o índice $d$ inicialmente foi favorável a $\mathrm{GC}(d=0,145$ ou 2,2 pontos de QI), mas após o treinamento o índice se torna favorável a $\mathrm{GE}(d=-0,51$ ou 7,6 pontos de QI)

Nos testes de inteligência cristalizada e de desempenho escolar não se observa maiores benefícios para GE. 
Efeito do Treinamento em GC e GE, Comparando QI Médio e Alto

Com a finalidade de investigar o efeito do treinamento considerando apenas as crianças de nível intelectual médio e alto, novamente se conduziu uma análise de variância de medidas repetidas intra e inter grupo (Tabela 3 ).

De forma geral, não foram encontrados efeitos estatisticamente significativos do treinamento para nenhuma das medidas consideradas utilizando apenas as crianças de nível intelectual alto e médio para as análises. Os resultados evidenciaram não haver efeito de tempo intra-grupo nos resultados do teste Raven $(\Lambda=1,000 ; F=0,000 ; p=0,995$; $\eta 2=0,000)$. Não houve, também, efeitos diferenciados entre os grupos $(p>0,05)$. No TNVRI, os resultados encontrados mostraram não haver efeito de tempo intra-grupos $(\Lambda=0,954 ; F=0,436 ; p=0,526 ; \eta 2=0,046)$. Não houve, também, efeitos diferenciados entre os grupos ( $p>$ $0,05)$. Já para a CEPA, os resultados evidenciaram haver diferenças intra-grupos $(\Lambda=0,300 ; F=16,316 ; p=0,005$; $\eta 2=0,700)$, ou seja, as médias de desempenho se alteraram do pré-teste para o pós-teste para cada grupo (GE e GC) separadamente. $\mathrm{O}$ mesmo aconteceu para o TDE $(\Lambda=$ $0,358 ; F=17,959 ; p=0,002 ; \eta 2=0,642)$. Contudo, não foram encontradas diferenças significativas entre os grupos $(p>0,05)$ tanto para a bateria CEPA quanto para o TDE.

Quando se considera o índice $d$, observa-se o mesmo padrão anterior, isto é, nos testes de inteligência fluida parece ocorrer maior influência do treinamento cognitivo. Assim, no teste Raven a diferença favorável a GC ( $d$ $=0,175$ ou 2,6 pontos de QI) se reduz após treinamento cognitivo para $d=0,093$ (ou 1,4 pontos de QI). No TNVRI, a diferença favorável inicialmente a GE $(d=-0,148$ ou 2,2 pontos de QI) se torna maior após o treinamento cognitivo $(d=-0,5$ ou 7,5 pontos de QI). Contudo, nos testes de inteligência cristalizada e desempenho escolar, a diferença entre pré e pós-teste aumenta a favor de GC.

Tabela 3

Médias e Desvios-Padrões nos Testes Raven, TNVRI, CEPA e TDE, Antes e Depois do Treinamento para as Crianças de QI Médio e Alto $(N=12)$

\begin{tabular}{|c|c|c|c|}
\hline Grupo & Antes & Depois & $n$ \\
\hline \multicolumn{4}{|c|}{ Raven } \\
\hline Controle & $39,3(6,8)$ & $39,6(9,2)$ & 6 \\
\hline Experimental & $38,0(8,0)$ & $38,8(8,0)$ & 6 \\
\hline & $d=0,175$ & $d=0,093$ & \\
\hline \multicolumn{4}{|c|}{ TNVRI } \\
\hline Controle & $43,0(6,8)$ & $41,2(5,6)$ & 6 \\
\hline Experimental & $43,8(3,4)$ & $44,1(6,0)$ & 6 \\
\hline & $d=-0,148$ & $d=-0,5$ & \\
\hline \multicolumn{4}{|c|}{ CEPA TOTAL } \\
\hline Controle & $38,3(12,4)$ & $59,2(12,2)$ & 6 \\
\hline Experimental & $37,0(11,7)$ & $45,8(9,0)$ & 6 \\
\hline & $d=0,11$ & $d=1,25$ & \\
\hline \multicolumn{4}{|c|}{ TDE } \\
\hline Controle & $102,1(15,6)$ & $113,5(9,2)$ & 6 \\
\hline \multirow[t]{2}{*}{ Experimental } & $99,5(8,9)$ & $109,0(12,0)$ & 6 \\
\hline & $d=0,21$ & $d=0,421$ & \\
\hline
\end{tabular}

Nota. *Os desvios-padrões estão entre parênteses; a bateria CEPA total representa a somatória dos escores dos testes de Fluência, Sinônimos, Cálculos e Séries Numéricas.

\section{Discussão}

O presente estudo objetivou verificar a eficácia de um programa de memória de trabalho para melhoramento cognitivo de crianças. Em primeiro lugar, considerando a amostra total de crianças (todos os níveis intelectuais), análises de variância de medidas repetidas e índices $d$ mostraram não houver diferenças estatisticamente signi- ficativas entre GC e GE após o treinamento. Não obstante, tendências interessantes emergiram. Antes do treinamento cognitivo, o grupo controle apresentava pontuações levemente superiores em todas as medidas cognitivas utilizadas. Após o treinamento, a diferença entre os grupos GE e GC (favorável a GC) caiu cerca de 1 a 3 pontos de QI para as medidas de inteligência fluida (Raven e TNVRI), embora o grupo controle tenha continuado superando 
Mansur-Alves, M., Flores-Mendoza, C. \& Tierra-Criollo, C. J. (2013). Evidências Preliminares da Efetividade do Treinamento Cognitivo para Melhorar a Inteligência de Crianças Escolares.

GE na medida de inteligência cristalizada (CEPA) e em desempenho escolar (TDE). Pesquisas que utilizam treinamento em memória de trabalho têm obtido resultados positivos em aumentar a inteligência fluida (Jaeggi et al., 2008; Klingberg, 2010; Klingberg et al., 2005; Klingberg et al., 2002; Nutley et al., 2011). Alguns estudos apontam que a MT contribui para um bom desempenho em diversas tarefas cognitivas complexas, como resolução de problemas e raciocínio, e que MT e inteligência seriam processos assentados nos mesmos circuitos neuronais, tais como o córtex parietal e pré-frontal (Ackerman et al., 2005; Jaeggi et al., 2011; Kane \& Engle, 2002). De acordo com Barnett e Ceci (2002), para que o treinamento ou o aprendizado em um processo/conteúdo possa ser transferido a outro é necessário que os elementos e os mecanismos subjacentes a um deles também estejam presentes no outro. Em se tratando da transferência do ganho de treinamento em MT para a inteligência cristalizada ( $\mathrm{G} c$ ), a literatura não é unânime. O estudo realizado por Alloway e Alloway (2009) apresenta evidências favoráveis à transferência do treino em MT para testes de Gc. Entretanto, Klauer e Phye (2008) encontraram resultados contrários aos apresentados pelos autores acima, mostrando que o treinamento em processos cognitivos fluidos não se transfere para medidas de inteligência cristalizada, uma vez que essas últimas estão relacionadas o armazenamento e o uso da memória de longo prazo, a qual está assentada em circuitos hipocampais. Os resultados encontrados no presente estudo seguem a tendência daqueles encontrados por Klauer e Phye (2008), ou seja, a não transferência do treinamento em MT para a medida de inteligência cristalizada. Há, não obstante, poucos estudos na literatura que consideram avaliação dos ganhos do treinamento medidas de inteligência fluida e cristalizada em um mesmo delineamento, conforme aquele encontrado no presente estudo. Contudo, outra hipótese parece ser igualmente viável. Se considerarmos que também não houve transferência dos ganhos de GE em inteligência fluida para o desempenho escolar e que as medidas de desempenho se assemelham às medidas de $\mathrm{G} c$, poderíamos pensar que não houve tempo suficiente para que os ganhos em $\mathrm{G} f$ fossem convertidos em aprendizado escolar (conhecimento). Essa hipótese nos parece atrativa, tendo em vista que os pós-testes foram realizados apenas em uma semana após o término do treinamento. Seria necessário que um segundo pós-teste fosse feito, com um intervalo mais longo após o treino, para verificar se os novos circuitos e processos adquiridos pelas crianças treinadas já estariam preparados para absorver maior informação do ambiente (Garlick, 2002). Ademais, o fato de o grupo controle ter realizado jogos informatizados, sendo que um deles (tetris) é reconhecido pelo impacto que possui no treinamento de circuitos atencionais (Haier, Siegel, Tang, Abel, \& Buchsbaum, 1992) acredita-se que pode ter sido responsável, em parte, pela reduzida diferença encontrada entre GC e GE. Ainda, conforme destaca Prins, Dovins, Ponsioen, Brink e van der Oord (2011), jogos de computador de maneira geral, dada à sua semelhança, aos videogames, despertam grande interesse em crianças, sendo extremamente motivadores. Os autores apresentam evidências de que, pelo menos para amostras clínicas, atividades de treinamento cognitivo que se assemelham mais videogames do que a tarefas clássicas de treinamento possuem maior eficácia para promover mudanças cognitivas, embora não se saiba exatamente quais os elementos do formato videogame contribuam para essa diferença.

O segundo ponto de interesse sobre o efeito do treinamento cognitivo se refere aos resultados encontrados quando são considerados, nas análises, os grupos de inteligência média/alta e média/baixa. Novamente, não foi encontrado efeito significativo do treinamento. Contudo, observa-se uma melhor resposta ao treinamento para o grupo de QI médio/ baixo do que para o de QI médio/alto, vista através da redução mais elevada da diferença inicial existente entre GE e GC. Para o grupo de QI médio/baixo, houve aumento do desempenho de GE nas medidas de inteligência fluida, ao passo que não foi observado especial ganho em inteligência fluida para o grupo de QI médio/alto. Esse resultado encontra respaldo parcial na literatura. Campbell et al. (2002), Herrnstein e Murray (1994) e Nisbett (2009) apontam que a maioria dos programas de intervenção cognitiva existentes foram concebidos para crianças de baixa inteligência ou com risco de atrasos no desenvolvimento. Esses autores destacam que tais programas objetivam suprir uma lacuna existente no desenvolvimento dessas crianças oferecendo estimulação ambiental adequada que possa acelerar o desenvolvimento cognitivo. Assim, pois, crianças de menor nível intelectual se beneficiariam mais dos programas de treinamento, uma vez que esses forneceriam a elas um ambiente de estimulação estruturado e dirigido. Por outro lado, conforme mostram Hager e Hasselhorn (1998), Haier (2006) e Herrnstein e Murray (1994) indivíduos mais inteligentes absorvem mais rápida e eficazmente os conteúdos que lhe são ministrados, apresentando ganho igual ou superior ao das crianças de menor inteligência. Os resultados encontrados no presente estudo confirmam que as crianças de QI médio/baixo se beneficiaram mais do treinamento do que as de QI médio/alto, mas não confirmam a presença de ganhos para o segundo grupo. Acredita-se que isso pode não ter ocorrido, porque ao se diminuir a exigência cognitiva das tarefas utilizadas para treinamento a fim de adequá-las às crianças de menor nível intelectual não se ofereceu desafios cognitivos suficientes para desequilibrar o sistema cognitivo das crianças mais inteligentes proporcionando melhora.

Não obstante, faz-se importante ressaltar que, de acordo com todas as considerações apontadas acima, poder-se-ia concluir que se foram encontrados benefícios do treinamento cognitivo, no presente estudo, eles o foram para as medidas de inteligência fluida e não para as de conhecimento e desempenho escolar. Embora sendo apenas uma tendência (não alcançando significância estatística), esses resultados trazem contribuições importantes para a área. Em primeiro lugar, não há estudos brasileiros que visam avaliar a eficácia de programas de treinamento cognitivo 
(em especial, de memória de trabalho) em adultos e em crianças. Assim, a iniciativa se faz pioneira. Em segundo lugar, mesmo os estudos internacionais que utilizam o treinamento em memória de trabalho não o fazem para crianças de diferentes níveis intelectuais. Nesse sentido, o presente trabalho também representa um avanço. Não obstante algumas contribuições alcançadas, algumas considerações sobre as limitações do estudo e propostas são necessárias.

A primeira ressalva a ser feita é a de que apesar do estudo contar com um design sofisticado (medidas $\mathrm{G} f \mathrm{e}$ Gc e controle de níveis de QI), ele é um estudo preliminar sobre intervenção em inteligência. E como tal deve ser visto. Tinha-se a intenção de comparar GC e GE para cada nível de inteligência individualmente. Entretanto, tal análise não foi possível, pois sendo a amostra total já bastante reduzida, a fragmentação da mesma em grupos menores careceria de sentido. Não se conhecem as razões de alguns pais não terem permitido o estudo dos seus filhos, mas a desistência pode ter gerado viés em nível comportamental (ex. filhos mais ou menos colaboradores), mas não viés em nível cognitivo uma vez que o presente estudo incluiu crianças de diferentes níveis intelectuais. A inclusão de um maior número de crianças em cada grupo intelectual possibilitaria o aparecimento de tendências mais claras de reais diferenças nos ganhos dos grupos. Portanto, novas replicações ou novos estudos com amostras maiores se torna necessário. Propõem-se, ainda, modificações na atividade realizada pelo grupo controle (mais motoras e sem envolvimento de jogos que demandem atenção cognitiva). Finalmente se recomenda realizar um estudo de pós-teste em intervalos maiores de tempo do que realizado no presente estudo (uma semana), e que por razões logísticas relacionadas ao calendário escolar não pôde ser feito. Acredita-se que essas modificações possam contribuir para superar as limitações apresentadas, trazendo informações importantes para o campo.

\section{Referências}

Ackerman, P. L., Beier, M. E., \& Boyle, M. O. (2005). Working memory and intelligence: The same or different constructs? Psychological Bulletin, 131(1), 30-60.

Alloway, T. P., \& Alloway, R. G. (2009). The efficacy of working memory training in improving crystallized intelligence. Nature Precedings. Retrieved January 17, 2011, from http:// precedings.nature.com/documents/3697/version/1

Associação Brasileira de Empresas de Pesquisa. (2003). Critério de Classificação Econômica Brasil-CCEB-Dados com base no levantamento socioeconômico 2000-IBOPE. Recuperado em 09 de outubro, 2010, em http//www.abep.org

Baddeley, A. (2003). Working memory: Looking back and looking forward. Nature Reviews: Neuroscience, 4, 829-839.

Barnett, S. M., \& Ceci, S. J. (2002). When and where do we apply what we learn? A taxonomy for far transfer. Psychological Bulletin, 128, 612-637.

Buschkuehl, M., \& Jaeggi, S.M. (2010). Improving intelligence: A literature review. Swiss Medical Weekly, 140(19-20), 266-272.
Campbell, F. A., Wasik, B. H., Pungello, E., Burchinal, B., Barbarin, O., Kainz, K., ...Ramey, C. T. (2002). Young adults outcomes from The Abecedarian and CARE early childhood educational interventions. Early Childhood Research Quarterly, 23(4), 452-456.

Centro Editor de Psicologia Aplicada. (2001a). Manual das Matrizes Progressivas de Raven - Escala Geral. Séries A, $B, C, D$ e $E$ (2. ed., F. Campos, Trad. \& Adaptação). Rio de Janeiro, RJ: Autor.

Centro Editor de Psicologia Aplicada. (2001b). Bateria Fatorial CEPA - Teste de Aptidões Especificas. Rio de Janeiro, RJ: Autor.

Chein, J. M., \& Morrison, A. B. (2010). Expanding the mind's workspace: Training and transfer effects with a complex working memory span task. Psychonomic Bulletin \& Review, 17(2), 193-199.

Colom, R. (2008). Nos limites da inteligência: É o ingrediente do êxito na vida? (C. Flores-Mendoza \& F. M. Franco, Trads.). São Paulo, SP: Vetor.

Colom, R., \& Flores-Mendoza, C. (2001). Inteligencia y memoria de trabajo: la relación entre factor $g$, complejidad cognitiva y capacidad de procesamiento. Psicologia: Teoria \& Pesquisa, 17, 37-47.

Colom, R., Quiroga, M. A., Shih, P. S., Martínez, K., Burgaleta, M., Martínez-Molina, A., ...Ramírez, I. (2010). Improvement in working memory is not related to increased intelligence scores. Intelligence, 38, 497-505.

Conboy, J. E. (2003). Algumas medidas típicas univariadas da magnitude do efeito. Avaliação Psicológica, 2, 145-158.

Conway, A. R. A., Kane, M. J., \& Engle, R. W. (2003). Working memory capacity and its relation to general intelligence. Trends in Cognitive Sciences, 7, 547-552.

Dantas, J. B. (1979). Efeitos de estimulação escolar na realização de crianças em vários estados nutricionais. Cadernos de Pesquisa, 29, 97-110.

Flores-Mendoza, C. E., Colom, R. B., Garcia, L. F., \& Castilho, A. V. (2001). Dificultades en el rendimiento escolar y la memoria de trabajo. Boletim de Psicologia, 1, 21-36.

Garlick, D. (2002). Understanding the nature of the general factor of intelligence: The role of individual differences in neural plasticity as an explanatory mechanism. Psychological Reviews, 109(1), 116-136.

Gray, R., \& McCormick, M. (2005). Early Childhood Intervention Programs in the US: Recent advances and future recommendations. The Journal of Primary Prevention, 26(3), 259-275.

Hager, W., \& Hasselhorn, M. (1998). The Effectiveness of the Cognitive Training for children from a differential perspective: A metaevaluation. Learning and Instruction, 8(5), 411-438.

Haier, R. (2006). Teoria Biológica da Inteligência. In C. Flores-Mendoza \& R. Colom (Orgs.), Introdução à Psicologia das Diferenças Individuais (pp. 131-142). Porto Alegre, RS: Artmed.

Haier, R. J., Siegel, B., Tang, C., Abel, L., \& Buchsbaum, M. S. (1992). Regional glucose metabolic changes after learning a complex visuospatial/motor task: A positron emission tomography study. Brain Research, 570, 134-143.

Halpern, D. (1997). Sex differences in intelligence: Implications for Education. American Psychologist, 52(10), 1091-1102.

Herrnstein, R. J., \& Murray, C. (1994). The Bell Curve: Intelligence and Class Structure in American Life. New York: Free Press. 
Mansur-Alves, M., Flores-Mendoza, C. \& Tierra-Criollo, C. J. (2013). Evidências Preliminares da Efetividade do Treinamento Cognitivo para Melhorar a Inteligência de Crianças Escolares.

Holmes, J., Gathercole, S. E., \& Dunning, D. L. (2009). Adaptive training leads to sustained enhancement of poor working memory in children. Developmental Science, 12(4), 9-15.

Jaeggi, S. M., Buschkuehl, M., Jonides, J., \& Perrig, W. J. (2008). Improving fluid intelligence with training on working memory. Proceedings of the National Academy of Sciences, 105(19), 6829-6833.

Jaeggi, S. M., Buschkuehl, M., Jonides, J., \& Shah, P. (2011). Short- and long-term benefits of cognitive training. Proceedings of the National Academy of Sciences, 108(25), 1008110086.

Jensen, A. R. (1981). Raising the IQ: The Ramey and Haskins Study. Intelligence, 5, 29-40.

Kane, M. J., \& Engle, R. W. (2002). The role of prefrontal cortex in working-memory capacity, executive attention, and general fluid intelligence: An individual differences perspective. Psychonomic Bulletin and Review, 9, 637-671.

Klauer, K. J., \& Phye, G. D. (2008). Inductive reasoning: A training approach. Review of Educational Research, 78(1), 85-123.

Klingberg, T. (2010). Training and plasticity of working memory. Trends in Cognitive Sciences, 14(7), 317-324.

Klingberg, T., Fernell, E., Olesen, P. J., Johnson, M., Gustafsson, P., Dahlstrom, K., ...Westerberg, H. (2005). Computerized training of working memory in children with ADHD - A randomized, controlled trial. Journal of American Academic Child Adolescent Psychiatry, 44(2), 177-186.

Klingberg, T., Forssberg H., \& Westerberg, H. (2002). Training of working memory in children with ADHD. Journal of Clinical Experimental Neuropsychology, 24(6), 781-791.

Kyllonen, P. C., \& Christal, R. E. (1990). Reasoning ability is (little more than) working-memory capacity?! Intelligence, 14, 389-433.

Lubinski, D. (2004). Introduction to the Special Section on Cognitive Abilities: 100 Years After Spearmen's (1904) “"General Intelligence', Objectively Determined and Measured". Journal of Personality and Social Psychology, 86(1), 96-111.

Miller, E. K., \& Cohen, J. D. (2001). An integrative theory of pre-frontal cortex function. Annual Review of Neuroscience, 24, 167-202.

Moody, D. E. (2009). Can intelligence be increased by training on a task of working memory? Intelligence, 37, 327-328.

Neisser, U., Boodoo, G., Bouchard, T. J., Boykin, A. W., Brody, N., Ceci, S. J., ...Urbina, S. (1996). Intelligence: Knows and Unknowns. American Psychologist, 51(2), 77-101.

Nisbett, R. E. (2009). Intelligence and How To Get It: Why Schools and Cultures Count. New York: W. W. Norton \& Company.

Nutley, S. B., Soderqvist, S., Bryde, S., Thorell, L. B., Humphreys, K., \& Klingberg, T. (2011). Gains in fluid intelligence after training non-verbal reasoning in 4-year-old children: A controlled, randomized study. Developmental Science, 14(3), 591-601.

Pasquali, L. (2005). Teste Não-Verbal de Raciocinio Infantil (TNVRI). Livro de Instruções. São Paulo, SP: Vetor.

Prins, P. J. M., Dovis, S., Ponsioen, A., Brink, E. T., \& van der Oord, S. (2011). Does computerized working memory training with game elements enhance motivation and training efficacyin children with ADHD? Cyberpsychology, Behavior, and Social Networking, 14(3), 115-122.

Ramey, C. T., \& Ramey, S. L. (1998). Early intervention and early experience. American Psychologist, 53(2), 109-120.

Shipstead, Z., Redick, T. S., \& Engle, R. W. (2010). Does working memory training generalize? Psychologica Belgica, 50(34), 245-276
Stein, L. M. (1994). TDE - Teste de Desempenho Escolar: Manual para aplicação e interpretação. São Paulo, SP: Casa do Psicólogo.

Strenze, T. (2007). Intelligence and socioeconomic success: A meta-analytic review of longitudinal research. Intelligence, 35, 401-426.

Thompson-Schill, S. L., Ramscar, M., \& Chrysikou, E. G. (2009). Cognition without control: When a little frontal lobe goes a long way. Current Directions in Psychological Science, 18(5), 259-263.

Thorell, L. B., Lindqvist, S., Bergman, N. S., Bohlin, G., \& Klingberg, T. (2009). Training and transfer effects of executive functions in preschool children. Developmental Science, 12(1), 106-113.

Wechsler, D. (2002). WISC-III: Escala de Inteligência Wechsler para Crianças: Manual (3. ed.). São Paulo, SP: Casa do Psicólogo. 


\section{Anexo}

Fórmula para o Cálculo do Índice d de Cohen

O índice $d$, ou diferença Cohen, constitui diferenças entre os grupos expressas em unidades de desvios padrões. Este índice permite fazer comparações entre resultados vindos da aplicação de diferentes instrumentos de medida psicológica (Conboy, 2003). A fórmula para tal cálculo é:

(1)

$$
\begin{aligned}
& \frac{\mathrm{X}_{1}-\mathrm{X}_{2}}{\text { Spooled }} \\
& s=\sqrt{\frac{\left(n_{1}-1\right) s_{1}^{2}+\left(n_{2}-1\right) s_{2}^{2}}{n_{1}+n_{2}-2}}
\end{aligned}
$$

Onde:

na fórmula (1) $\mathrm{X}_{1}$ e $\mathrm{X}_{2}$ são as médias das amostras 1 e 2 respectivamente. $S$ pooled é a variância agrupada e é calculada por meio da fórmula (2), onde $n_{1}$ é o tamanho da amostra do grupo $1 ; n_{2}$ é o tamanho da amostra do grupo $2 ; \mathrm{S}_{1}$ é o desvio-padrão do grupo 1 e $\mathrm{S}_{2}$ é o desvio-padrão do grupo 2 . 